\title{
Screening Patients with Severe Mental Illness for Metabolic Syndrome: A Quality Improvement Project by a Mobile Medication Nursing Program
}

\section{Cynthia Eldredge RN ${ }^{1 *}$, Noreen Fredrick DNP RN ${ }^{1}$, Bruce Gnesda RN ${ }^{1}$, Mary-Catherine Bohan LCSW $^{1}$, Frank Ghinassi ${ }^{1,2}$ and Roy Chengappa KN ${ }^{1,2}$}

${ }^{1}$ Western Psychiatric Institute and Clinic, University of Pittsburgh Medical Center, Pittsburgh, PA, USA

${ }^{2}$ University of Pittsburgh School of Medicine, Pittsburgh, PA, USA

Corresponding author: Cynthia Eldredge RN, Mobile Medication Program Coordinator, Western Psychiatric Institute and Clinic, University of Pittsburgh Medical Centre, 3811 O'Hara Street, Pittsburgh, Pennsylvania 15213-2593, USA, Tel: 412-204-9019; Fax: 412-204-9132, E-mail: eldrcr@upmc.edu

Rec date: April 08, 2016; Acc date: April 29, 2016; Pub date: May 05, 2016

Copyright: (C) 2016 Eldredge RNC, et al. This is an open-access article distributed under the terms of the Creative Commons Attribution License, which permits unrestricted use, distribution, and reproduction in any medium, provided the original author and source are credited.

Citation: Eldredge RNC, Fredrick DNPRNN, Gnesda RNB, et al. Screening Patients with Severe Mental Illness for Metabolic Syndrome: A Quality Improvement Project by a Mobile Medication Nursing Program. 2016, 2:2.

\section{Abstract}

Background: Persons with serious mental illnesses (SMI) die 25 years younger than the general population due to risks associated with smoking, excess weight, poor diet and sedentary life styles. Screening for metabolic syndrome in SMI patients and communicating results can reduce this risk.

Methods and Findings: This quality improvement initiative provided training to measure components of metabolic syndrome, and to document and communicate the results to physicians. All 62 clients were screened during a 3-month period; 20 (32.3\%) met full criteria, 36 individuals met at least one or two individual criteria of metabolic syndrome; 6 did not. These visits were done inhome, thus blood glucose and lipid values were not available, but however, those receiving treatments for diabetes mellitus; dyslipidemia; taking fish oil or niacin for low HDL cholesterol were enumerated. Conclusions: Results of the screening of SMI patients for metabolic syndrome were successfully communicated to physicians to initiate appropriate interventions.

Keywords: Communication metabolic syndrome; Physicians; Serious mental illness

\section{Introduction}

The life expectancy of individuals with serious mental illness (SMI) is nearly 25 years less than the general population [1]. Risk factors include a poor diet, sedentary lifestyles, high rates of smoking, and excessive weight gain and metabolic concerns such as diabetes mellitus or dyslipidemia associated with some psychotropic medications [2-5]. Inspite of exhortations from professional organizations [6] and expert panels [7,8] to monitor for these modifiable risks, studies have shown mixed results for such monitoring [9-11]. These risk factors can lead to morbidity and mortality from cardiovascular disorders, diabetes mellitus, and essential hypertension among others $[12,13]$. Western Psychiatric Institute and Clinic (WPIC) of the University of Pittsburgh's Medical Center is an academic tertiary care provider of psychiatric services, more specifically, its Comprehensive Recovery Services (CRS) service line provides treatment and support to over 3000 individuals with SMI. In response to this alarming statistic, discussions among the WPIC and CRS leadership led to targeting the assessment of the metabolic syndrome among the SMI patients as a first step to address this issue. The plan was to assess and document if patients with SMI met criteria (or not) for the metabolic syndrome or its individual components. Initially, this was implemented in two large ambulatory clinics [14]. It was then expanded to other community based programs, one of which is the Mobile Medication Program. The Mobile Medication Program was created in anticipation of closure of a regional state psychiatric hospital and is comprised of psychiatric nurses who serve individuals with SMI in their homes. These individuals are at high risk for both psychiatric and physical health illnesses, as well as unplanned psychiatric and medical hospitalizations.

\section{Methods}

An electronic health record tool for screening and monitoring the metabolic syndrome indices in persons with SMI within the larger context of WPIC is detailed in Nash et al. Building on the work of Nash et al., the CRS leadership, in collaboration with information technology staff developed an electronic health record (EHR) Metabolic Syndrome tool which tracks height, weight, BMI (body mass index), waist circumference, and blood pressure, glucose, and lipid levels. This tool also incorporates whether a patient is receiving insulin/oral hypoglycaemic agents, or statins or other agents (including fish oil and niacin) for dyslipidaemia and/or antihypertensive medications. Once recorded, the programming in the electronic tool computes if an individual patient meets full criteria for metabolic syndrome or not, and an alert is flashed if positive. The health care professional documenting the 
encounter can then write a narrative text on action steps; examples: counselling, referrals, communication to the primary care physician and/or psychiatrist, etc. Initially available only to the physicians, the tool was later developed as a stand-alone tool that could be used by other health care professionals, and was incorporated for use by nurses in the Mobile Medication Program.

The goals of the assessment within the mobile medication program were to a) screen $95 \%$ of the entire caseload for Metabolic Syndrome, i.e. the anthropometric measures (height, weight, body mass index, blood pressure and waist circumference), and record any treatment for diabetes, dyslipidaemia (including niacin or fish oil for low HDL) and/or essential hypertension and b) communicate $95 \%$ of metabolic syndrome treatment alerts to a primary care physician and/or psychiatrist to take next steps as clinically appropriate. During a 3-month period, a total of 62 individuals served in the Mobile Medication program (100\%) were screened. The Mobile Medication program nurses were trained on reliably measuring individual components of Metabolic Syndrome. Each nurse was equipped with rolling suitcases, weighing scales, wrist sphygmomanometers, and measuring tapes for waist circumference. We established a protocol for communicating positive treatment alerts to physicians, either an identified primary care doctor or the treating psychiatrist or both by emailing or calling the physician's office. Communication efforts were documented in the metabolic syndrome tool within the electronic health record. As the project progressed, the Mobile Medication program nurses identified and assisted in overcoming operational barriers to collecting and acting on screening results in the field. For instance, one primary stumbling block was the inability to complete the screen in its entirety without having access to blood work results (examples: blood glucose or lipid values). This concern was mitigated somewhat by checking known medications in the electronic health record or reviewing medications that patients were receiving, examples: statins or anti-hypertensive or anti-diabetic agents, which even without the actual laboratory results would suffice to trigger a positive treatment alert.

\section{Results}

62 clients screened for metabolic syndrome, 20 met full criteria (i.e. 3 or more individual components) or $32.3 \%, 36$ individuals met criteria for at least one or two individual components of the metabolic syndrome and 6 did not. A further breakdown of the 36 individuals who did not meet full criteria but had one or two individual components revealed: 17 met criteria for two components and 19 met criteria for one component of metabolic syndrome. Moreover, 24 of the 36 individuals were classified as obese (the EHR was also programmed to trigger an alert, based on computation of the BMI status) and their individual physicians were notified. The screening also alerted the nurses to those who were overweight but not necessarily obese (again based on BMI status). As the project progressed, nurses began to communicate the presence of positive Metabolic Syndrome treatment alerts and by 3-months, $100 \%$ of the alerts had been communicated. At the time of writing (2016), the nurses continue monthly monitoring of the metabolic syndrome of patients enrolled in this program, and continue to communicate these physical health concerns to healthcare providers, and encourage patients to keep medical appointments to manage these medical conditions appropriately.

\section{Discussion}

The two primary goals of this quality improvement (QI) project were exceeded $100 \%$ of the patients in the program were screened, and $100 \%$ of the results were communicated to physicians. This QI project reflects a successful attempt to change clinician practice and behaviour utilizing a low-tech, low-cost, and pragmatic approach with administrative, clinician and patient "buy-in". The primary stumbling blocks were the inability to complete the screen in its entirety without having access to blood work results during in-home visits. These concerns were mitigated to an extent by checking medications used to treat diabetes, high or low cholesterol (i.e. LDL vs HDL) and hypertension, and by recording anthropometric measures into the EHR tool which in turn triggered treatment alerts. Anecdotally, we noted a change in the nurse's focus leading to increased interest and discussions on diet and nutrition, portion control, food choices, weight management, blood pressure control, sodium intake, exercise and smoking cessation. In one study, nurses provided educational information at two clinics to improve the knowledge base of mental health counsellors regarding metabolic syndrome, and this intervention resulted in improved screening of BMI which in turn lead to referrals of SMI patients to physicians for weight loss treatments [15]. A more recent review concluded that small sample size studies dominate the literature for screening and non-pharmacological interventions for metabolic syndrome in SMI patients and those more definitive studies are needed to guide nursing practice [16].

While some antipsychotic medications (examples: clozapine and olanzapine) are more likely to induce weight gain and insulin resistance than others, given that several SMI patients live sedentary life styles, smoke cigarettes, and eat unhealthily, screening all patients regardless of medications for metabolic syndrome can target these modifiable risks. We intend to continue expanding this QI project to the next critical phase, i.e. continued interventions for weight loss, smoking cessation, life-style and medication management of cardiac, diabetes, dyslipidemia, and other disorders. We hope such targeted efforts by health care providers will help regain some of the 25 years of life lost in this highly vulnerable patient population.

\section{Acknowledgements}

The authors presented a condensed earlier version poster at the 2013 Dr. Loren Roth UPMC Quality and Patient Safety Symposium, "The Leader in Me", held on October 30, 2013 at the Herberman Conference Center at UPMC Cancer Pavilion, 
UPMC Shadyside. The authors thank Joan Spinogatti for data entry, collaboration among authors, assisting in submission of the manuscript; Deborah DiLeonardo RN, Christopher Johnson RN, Barbara Jones RN, Gloria Pollard RN, Susan Quinn RN, the original team members, Ellie Medved MSN and Ken Nash, MD, MMM for executive support and encouragement, and Kalyani Gopalan MSHCPM, MHA for database management and analyses.

\section{Potential Conflicts of Interest}

The authors have no financial disclosures with regards to this study.

\section{References}

1. Parks J, Svendsen D, Singer P, Foti ME (2006) Morbidity and Mortality in People with Serious Mental Illness. Sixteen-State Study on Mental Health Performance Measures. National Association State Mental Health Program Directors.

2. Allison DB, Casey DE (2001) Antipsychotic-induced weight gain: a review of the literature. J Clin Psychiatry 7: 22-31.

3. Lasser K, Boyd JW, Woolhandler S, Himmelstein DU, McCormick $D$, et al. (2000) Smoking and mental illness: A population-based prevalence study. JAMA 284: 2606-2610.

4. McEvoy JP, Meyer JM, Goff DC, Nasrallah HA, Davis SM, et al. (2005) Prevalence of the metabolic syndrome in patients with schizophrenia: baseline results from the Clinical Antipsychotic Trials of Intervention Effectiveness (CATIE) schizophrenia trial and comparison with national estimates from NHANES III. Schizophr Res 80: 19-32.

5. Correll CU, Frederickson AM, Kane JM, Manu P (2008) Equally increased risk for metabolic syndrome in patients with bipolar disorder and schizophrenia treated with second-generation antipsychotics. Bipolar Disord 788-97.

6. American Diabetes Association, American Psychiatric Association, American Association of Clinical Endocrinologists, North American Association for the Study of Obesity (2004) Consensus development conference on antipsychotic drugs and obesity and diabetes. J Clin Psychiatry 65: 267-272
7. Marder SR, Essock SM, Miller AL, Buchanan RW, Casey DE, et al. (2004) Physical health monitoring of patients with schizophrenia. Am J Psychiatry 161: 1334-49.

8. Usher K, Foster K, Park T (2006) The metabolic syndrome and schizophrenia: the latest evidence and nursing guidelines for management. J Psychiatr Ment Health Nurs 6: 730-4.

9. Weissman EM, Zhu CW, Schooler NR, Goetz RR, Essock SM (2006) Lipid monitoring in patients with schizophrenia prescribed second-generation antipsychotics. J Clin Psychiatry 67: $1323-6$

10. Morrato EH, Newcomer JW, Kamat S, Baser O, Harnett J, et al. (2009) Metabolic screening after the American Diabetes Association's consensus statement on antipsychotic drugs and diabetes. Diabetes Care 6: 1037-42.

11. Haupt DW, Rosenblatt LC, Kim E, Baker RA, Whitehead R, et al. (2009) Prevalence and predictors of lipid and glucose monitoring in commercially insured patients treated with secondgeneration antipsychotic agents. Am J Psychiatry. 166: 345-53.

12. Colton CW, Manderscheid RW (2006) Congruencies in increased mortality rates, years of potential life lost, and causes of death among public mental health clients in eight states. Prev Chronic Dis 2: A42.

13. Kupfer DJ (2005) The increasing medical burden in bipolar disorder. JAMA 2930: 2528-30.

14. Nash K, Ghinassi F, Brar JS, Alam A, Bohan MC, et al. (2013) The Development and Implementation of an Electronic Health Record Tool for Monitoring Metabolic Syndrome Indices in Patients with Serious Mental Illness. Clin Schizophr Relat Psychoses.

15. Arms T, Bostic T, Cunningham P (2014) Educational intervention to increase detection of metabolic syndrome in patients at community mental health centers. J Psychosoc Nurs Ment Health Serv 52: 32-6.

16. Richards L, Batscha CL, McCarthy VL (2016) Lifestyle and Behavioral Interventions to Reduce the Risk of Metabolic Syndrome in Community-Dwelling Adults with Serious Mental Illness: Implications for Nursing Practice. J Psychosoc Nurs Ment Health Serv 54: 46-55. 